JOURNAL OF SYNCHROTRON RADIATION

ISSN 1600-5775

Received 31 March 2020

Accepted 26 June 2020

Edited by I. Lindau, SLAC/Stanford University, USA

Keywords: RIXS; X-ray spectroscopy; $h v^{2}$-spectrometer; core-hole lifetime; charge-neutral excitation.
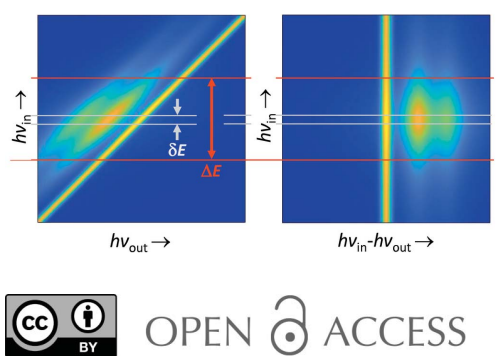

\section{$h v^{2}$-concept breaks the photon-count limit of RIXS instrumentation}

\author{
Ke-Jin Zhou, ${ }^{\mathrm{a} *}$ Satoshi Matsuyama ${ }^{\mathrm{b}}$ and Vladimir N. Strocov ${ }^{\mathrm{c} *}$ \\ ${ }^{\mathbf{a}}$ Diamond Light Source, Harwell Campus, Didcot OX11 ODE, United Kingdom, ${ }^{\mathbf{b}}$ Department of Precision \\ Science and Technology, Graduate School of Engineering, Osaka University, 2-1 Yamada-oka, Suita, Osaka, \\ Japan, and 'Swiss Light Source, Paul Scherrer Institute, 5232 Villigen-PSI, Switzerland. \\ *Correspondence e-mail: kejin.zhou@diamond.ac.uk, vladimir.strocov@psi.ch
}

Upon progressive refinement of energy resolution, the conventional resonant inelastic X-ray scattering (RIXS) instrumentation reaches the limit where the bandwidth of incident photons becomes insufficient to deliver an acceptable photon-count rate. Here it is shown that RIXS spectra as a function of energy loss are essentially invariant to their integration over incident energies within the core-hole lifetime. This fact permits RIXS instrumentation based on the $h v^{2}$-concept to utilize incident synchrotron radiation over the whole core-hole lifetime window without any compromise on the much finer energy-loss resolution, thereby breaking the photon-count limit.

\section{Introduction}

Synchrotron-radiation-based resonant inelastic X-ray scattering (RIXS) is one of the most advanced spectroscopic techniques giving access to the whole spectrum of chargeneutral excitations in condensed and soft matter, ranging from charge transfer and orbital excitations at its high-energy end of a few $\mathrm{eV}$ to magnon, phonon and many exotic elementary excitations at its low-energy end extending ultimately down to a few meV (Braicovich et al., 2010; La Tacon et al., 2011; Schlappa et al., 2012; Ghiringhelli et al., 2012; Chaix et al., 2017; Hepting et al., 2018; Arpaia et al., 2019). High energy resolution is a prerequisite of the RIXS experiment in pursuit of the progressively refining energy scale of these excitations.

During the last decade, RIXS instrumentation has seen enormous progress, with its state-of-the-art resolving power now approaching 50000. The increase comes on both the incident photon side, defined by the beamline energy resolution, and on the outgoing photon side, defined by the spectrometer energy resolution. At a certain stage of the resolution improvement, however, the incident photon-energy bandwidth reduces so much that the total number of photons delivered to the sample becomes insufficient for the accumulation of the RIXS signal within any reasonable acquisition time. We call this situation the photon-count limit of RIXS instrumentation. It cannot be circumvented on the route of the conventional RIXS instrumentation, where the resolution improvement inevitably reduces the utilized bandwidth of incident X-ray photons.

A way to break through the photon-count limit can be envisaged by bringing together insights into the fundamental physics of the RIXS process and novel instrumental concepts. On the physics side, the ability of RIXS to detect elementary excitations is due to the intermediate core-hole state. For instance, spin-flip excitations at the $L$-edge in many transition 
metal oxides are allowed thanks to the strong spin-orbit (LS) coupling of the $2 p$ core-hole state. Importantly, the intermediate state is naturally broadened due to the core-hole lifetime. Although the spectrum of the elementary excitations observed in RIXS critically depends on the incident energy involving different intermediate states, one can nevertheless expect that this spectrum stays constant as long as the incident energy is kept within the lifetime broadening of the selected intermediate state.

On the instrumental side, a way to utilize the extended bandwidth of the incident-photon energy $\left(h v_{\text {in }}\right)$ without any compromise on resolution in the outgoing-photon energy ( $h v_{\text {out }}$ ) can be realized with the recently suggested $h v^{2}$-concept of the RIXS spectrometer (Strocov, 2010). The line focus of light from the focal plane of the beamline monochromator, dispersed in $h v_{\text {in }}$ in the vertical direction, is imaged by refocusing optics onto the sample. The spectrometer uses a focusing mirror in its vertical (imaging) plane to refocus the image from the sample onto the position-sensitive detector, resolving the scattered light in $h v_{\text {in }}$. At the same time, a cylinder variable-line-spacing (VLS) grating operating in the horizontal (dispersive) plane of the spectrometer disperses and focuses the scattered light onto the detector, resolving it in $h v_{\text {out }}$. In this way the RIXS intensity is acquired as a twodimensional (2D) image in the $h v_{\text {in }}$ and $h v_{\text {out }}$ coordinates detected simultaneously (Strocov, 2010). Obviously, this concept implies sample homogeneity within the line focus on the sample, which can be squeezed by the refocusing optics to $\sim 100 \mu \mathrm{m}$ and less. Later, Warwick et al. realized that the main factor limiting the $h v_{\mathrm{in}}$-acceptance and thus efficiency of this concept is the spatial extension of the light footprint on the sample along the $h v_{\text {in }}$-direction, and suggested to solve this problem by replacing the focusing mirror in the imaging plane of the spectrometer by a Wolter-type imaging optics (Warwick et al., 2014). Furthermore, the focal-plane inclination at the sample has been eliminated with an elliptical pre-mirror of the monochromator. The first project to realize this scheme, the double-dispersion QERLIN spectrometer at the Advanced Light Source, is presently nearing its completion (Chuang et al., 2016). Remarkably, a dramatic increase of the overall scattered X-ray intensity detected with such an imaging- $h v^{2}$ spectrometer of about two orders of magnitude compared with the conventional state-of-the-art RIXS spectrometers comes without any compromise on the energy-loss resolution.

Here, we demonstrate fundamental scientific grounds allowing a breakthrough of the photon-count limit of RIXS instrumentation towards a progressively refining energy resolution. We report ultra-high-resolution RIXS experiments on a prototypical binary oxide, $\mathrm{CoO}$, across the $\mathrm{Co}_{2} L_{3}$-edge, and demonstrate that the RIXS spectral line profile on the energy-loss scale stays invariant of $h v_{\text {in }}$ as long as it is kept within the core-hole lifetime broadening. Furthermore, we analyze the RIXS intensity distribution in the $\left(h v_{\text {in }}, h v_{\text {out }}\right)$ coordinates generated by the imaging $h v^{2}$ spectrometer and demonstrate a way of rendering it into the RIXS spectra over the whole $h v_{\text {in }}$-window of the core-hole lifetime, increasing the efficiency of the RIXS experiment by one to two orders of magnitude without compromising its resolution on the energyloss scale.

\section{RIXS spectra across the absorption line}

Ultra-high-resolution experiments to explore the sensitivity of RIXS spectral structures, as a function of $h v_{\text {out }}$, to the variation of $h v_{\text {in }}$ were performed at the I21-RIXS beamline at Diamond Light Source, UK (https://www.diamond.ac.uk/ Instruments/Magnetic-Materials/I21.html). Single-crystal $\mathrm{CoO}$ was selected for the study focused on the low-energy excitations at the $\mathrm{Co}_{3}$-edge. The sample was aligned with the surface normal (100) lying in the horizontal scattering plane. Co $L_{3}$-edge X-ray absorption spectroscopy (XAS) data were collected in the fluorescence yield. For RIXS measurements, linear $\sigma$-polarization was used. The total energy resolution $\mathrm{d} E$ at the full width at half-maximum (FWHM) was about $33 \mathrm{meV}$ at the Co $L_{3}$-edge. The grazing-incidence and scattering angles $\theta$ and $2 \theta$ were fixed to $20^{\circ}$ and $154^{\circ}$, respectively. All measurements were made at a temperature of $13 \mathrm{~K}$.

Fig. 1(a) displays the Co $L_{3}$-edge XAS. The fine structure is dominated by the multiplet effects of the intermediate corehole states of the $\mathrm{Co}^{2+}$ ion within its crystal-field symmetry. Two $h v_{\text {in }}$ regions were chosen (red and blue highlights) to excite RIXS in a step of $0.03 \mathrm{eV}$. The low-energy RIXS spectra in these regions, presented in Figs. $1(b)$ and $1(c)$, were obtained by summation over $h v_{\text {in }}$-intervals of different energy windows. For example, the spectrum denoted by $\pm 0.00 \mathrm{eV}$ means it was acquired at the central incident energies $h v_{\text {in }}=$ $777.14 \mathrm{eV}$ and $778.99 \mathrm{eV}$ of the two regions, while the spectrum denoted by $\pm 0.15 \mathrm{eV}$ is the average of the RIXS spectra excited with $h v_{\text {in }}$ from $-0.15 \mathrm{eV}$ to $+0.15 \mathrm{eV}$ relative to the central incident energies. A three-peak structure is clearly present in all RIXS spectra. The shoulder peak at zero energy results from the quasi-elastic scattering from the sample, and the second and third peaks at $\sim 60 \mathrm{meV}$ and $\sim 120 \mathrm{meV}$ are arguably dominated by the one-magnon and two-magnon excitations, respectively (Sarte et al., 2019).

It is noticeable that the line shape of the averaged RIXS spectra deviates more and more from that of the central RIXS spectrum as the $h v_{\text {in }}$-window widens. To quantify our analysis, we define $P=\left[I_{\text {ave }}(E)-I_{0}(E)\right] / I_{0}(E)$ as a measure of the amount of deviation, in which $I_{\text {ave }}(E)$ represents the RIXS spectra averaged over the $h v_{\text {in }}$-window and $I_{0}(E)$ stands for the RIXS spectrum excited by the central $h v_{\text {in }}$. The value of $P$, plotted in Figs. 1(d) and 1(e), shows a monotonic increase as the $h v_{\text {in }}$-window widens to $\pm 250 \mathrm{meV}$. We can define a threshold, below which the RIXS spectra are regarded as invariant, as $P=10 \%$. This corresponds to an $h v_{\mathrm{in}}$-integration window of about $\pm 0.24 \mathrm{eV}$ which is roughly the FWHM of the Co $L_{3}$ core-hole lifetime broadening $0.43 \mathrm{eV}$ (Krause \& Oliver, 1979).

In this way, we have shown that the RIXS spectra can be integrated in an $h v_{\text {in }}$-window of the core-hole lifetime while keeping the whole spectroscopic information. We note that our case of magnon excitations can be considered as one of the most critical on the $h v_{\text {in }}$-bandwidth, and high-energy-loss 

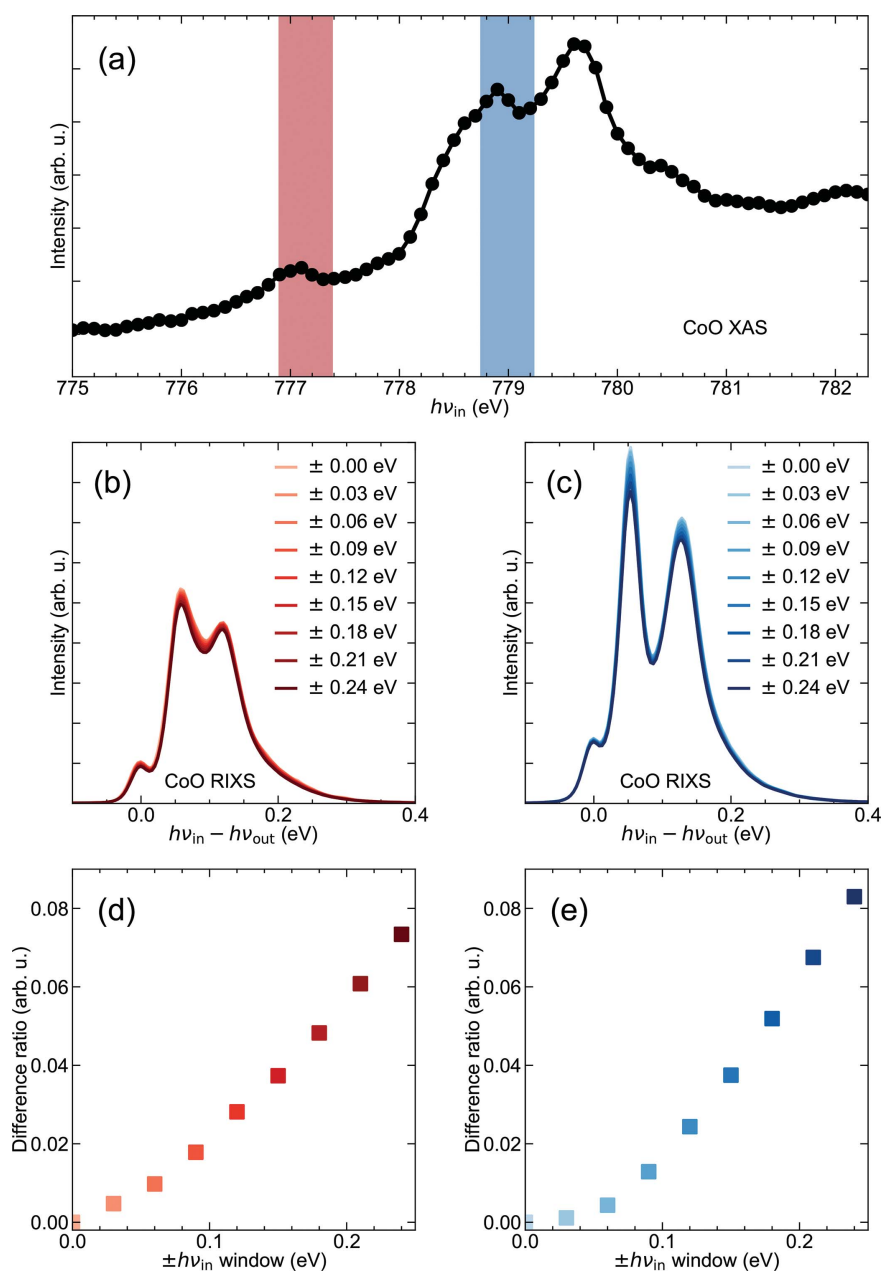

Figure 1

(a) The Co $L_{3}$-edge XAS spectrum collected using fluorescence yield. Red and the blue bars mark the energy windows where the RIXS spectra were acquired. $(b, c)$ Low-energy excitations in $\mathrm{CoO}$ as a function of the $h v_{\text {in }}$-integration window for the region marked in red $(b)$ and blue $(c)$ in $(a)$. $(d, e)$ Difference ratio $P$ between the integrated and central RIXS spectra as a function of the $h v_{\mathrm{in}}$-window (see the definition in the main text). excitations, for example, the orbital excitations, are typically less sensitive to it.

\section{Detection efficiency of the $h v^{2}$-concept}

We now demonstrate how the $h v^{2}$-concept translates the invariance of the RIXS spectra across the absorption line into a dramatic detection-efficiency increase. Fig. 2 schematizes an XAS spectral peak and corresponding RIXS intensity map, where the elastic peak stretching along the $h v_{\text {in }}=h v_{\text {out }}$ line is tracked by two Raman satellites at constant energy loss $h v_{\text {in }}-$ $h v_{\text {out }}$. The sought-for spectrum of RIXS intensity as a function of energy loss, delivered by the conventional spectrometer, is intrinsically integrated over $h v_{\text {in }}$ within the bandwidth $\delta E$ determined by the required combined beamline plus spectrometer energy resolution $\mathrm{d} E$ as $\delta E=\mathrm{d} E / \sqrt{2}$ (in the ideal case when the two contributions are balanced). In contrast, the full 2D map acquired with the imaging- $h v^{2}$ spectrometer allows us to obtain the RIXS spectrum by integrating along the $h v_{\text {in }}-h v_{\text {out }}$ direction within a much larger interval $\Delta E$ determined by the XAS peak-width. In this way, the $h v^{2}$ concept allows full utilization of a broad $h v_{\text {in }}$ bandwidth, realistically up to two orders of magnitude compared with the energy-loss resolution, which dramatically increases the detection efficiency without any sacrifice on the latter. It should be noted that the use of imaging optics in the refocusing and, as suggested by Warwick et al. (2014), spectrometer stages of the optical scheme ensures the invariance of energy resolution over the whole intercepted $h v_{\text {in }}$ range. We note that another concept that promises accepting a large $h v_{\text {in }}$ bandwidth without sacrificing the energy-loss resolution is the active-grating monochromator (AGM) and active-grating spectrometer (AGS) implemented at the Taiwan Photon Source (Lai et al., 2014). Such an instrument inherently integrates the RIXS intensity along the $h v_{\text {in }}-h v_{\text {out }}$ direction to deliver essentially a $1 \mathrm{D}$ spectrum, whereas the $h v^{2}$-concept delivers the full $2 \mathrm{D}$ map allowing integration either along constant $h v_{\text {in }}-h v_{\text {out }}$ to keep the energy resolution of the
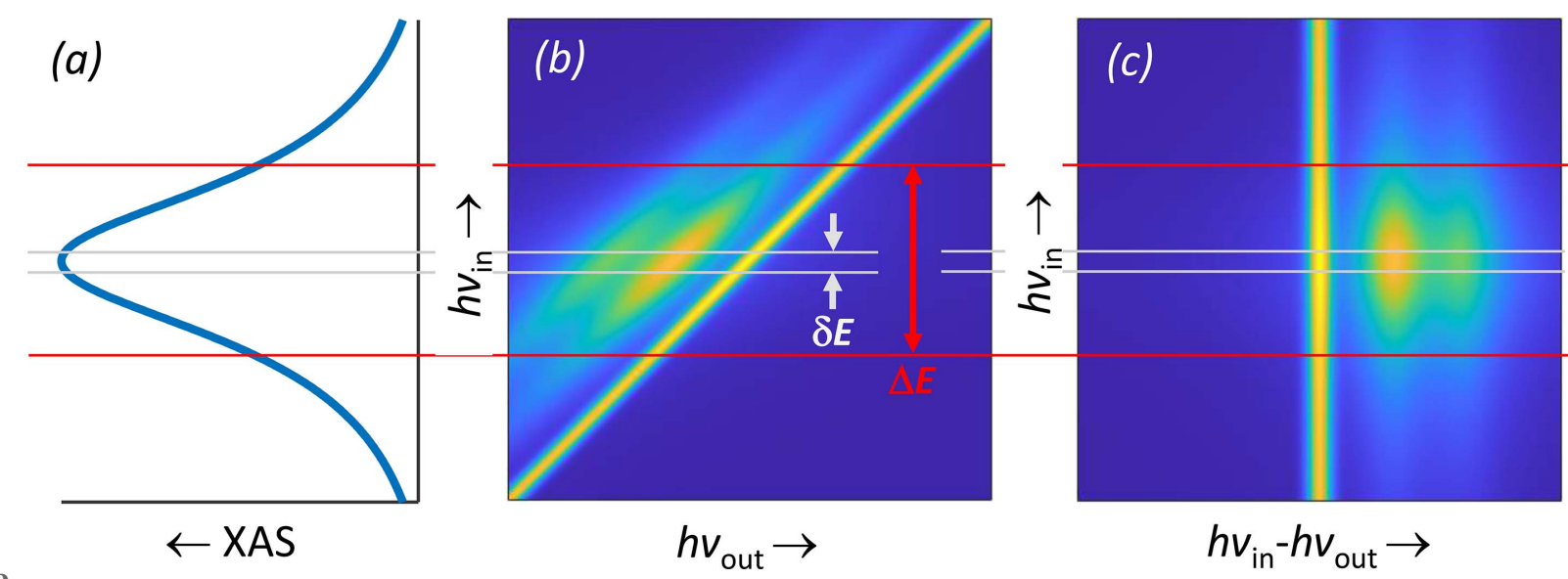

Figure 2

Schematic XAS spectrum (a) and the corresponding RIXS intensity map in the ( $\left.h v_{\text {in }}, h v_{\text {out }}\right)$ coordinates $(b)$ and rendered into the $\left(h v_{\text {in }}, h v_{\text {in }}-h v_{\text {out }}\right)$ coordinates $(c)$. Whereas the conventional RIXS spectrometer utilizes incident photons in a narrow bandwidth $\delta E$, the $h v^{2}$ one utilizes a much larger bandwidth $\Delta E$ of the XAS peakwidth. 


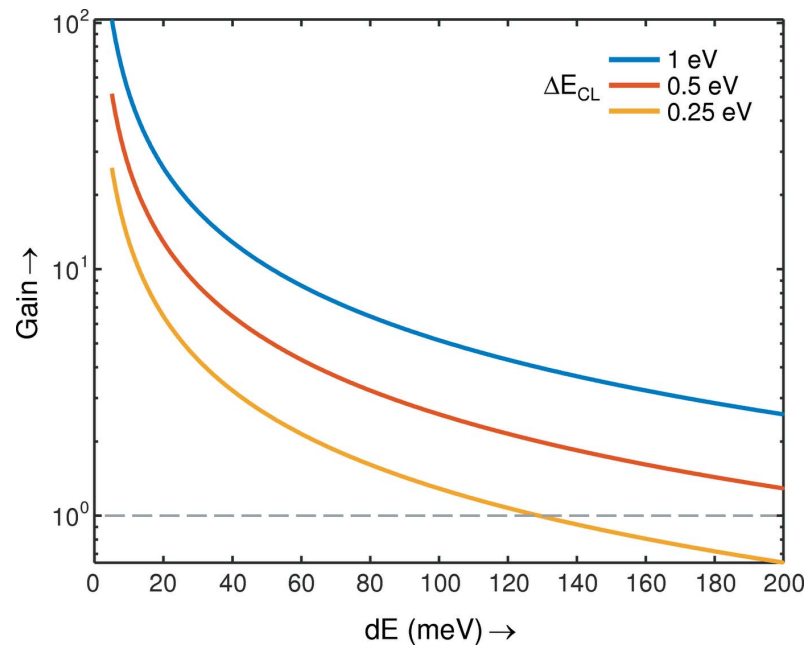

Figure 3

Intensity gain delivered by the imaging- $h v^{2}$ spectrometer relative to the conventional one as a function of energy-loss resolution for different core-level energy widths.

Raman peaks or along constant $h v_{\text {out }}$ to keep that of the fluorescence peaks.

Finally, we will assess the detection-efficiency gain delivered by the imaging- $h v^{2}$ spectrometer relative to the conventional RIXS spectrometer adopting the highest-transmission optical scheme consisting of a collecting mirror and a spherical VLSgrating (Ghiringhelli et al., 2006; Strocov et al., 2011). For the imaging- $h v^{2}$ spectrometer we assume a refocusing-optics stage employing two imaging pairs operating in the horizontal and vertical planes, and for the conventional one of a KirkpatrickBaez pair of ellipsoidal mirrors. The gain is defined essentially by the ratio of the FWHM core-level $\Delta E_{\mathrm{CL}}$, utilized by the former, to the beamline FWHM resolution, utilized by the latter. This ratio should be further reduced to account for the three additional mirrors in the imaging- $h v^{2}$ scheme compared with the conventional one [two additional reflections in the imaging-optics refocusing stage and one in the imaging stage of the spectrometer (Warwick et al., 2014; Chuang et al., 2016), a factor of $\sim 0.9$ at each] and for another factor of $\sim 0.9$ describing the average amplitude of the XAS peak within its FWHM relative to the peak value. The results are presented in Fig. 3 as a function of combined $\mathrm{d} E$ for three values of $\Delta E_{\mathrm{CL}}$. Obviously, the gain is proportional to $\Delta E_{\mathrm{CL}}$ and blows up with the resolution refinement. Even for the lowest $\Delta E_{\mathrm{CL}}=$ $250 \mathrm{meV}$, the imaging- $h v^{2}$ spectrometer overtakes the conventional one starting already from $\mathrm{d} E \simeq 115 \mathrm{meV}$, achieves a detection-efficiency advantage of $\sim 4$ at the currently standard $\mathrm{d} E \simeq 30 \mathrm{meV}$, and boosts this advantage in a singular manner with further refinement of $\mathrm{d} E$. These results clearly show the breakthrough of the photon-count limit towards progressive energy-resolution refinement. In free speech, it is really astonishing to see how all incident photons within a bandwidth, say, of $1 \mathrm{eV}$ build up a RIXS spectrum with a resolution of $10 \mathrm{meV}$.

We will now illustrate these considerations with our experimental example at the Co $L_{3}$-edge presented above in
Fig. 1, where the total resolution $\mathrm{d} E$ was $\sim 33 \mathrm{meV}$ at the incident X-ray beam bandwidth $\delta E \simeq 23 \mathrm{meV}$. The latter corresponds to an exit slit opening of $10 \mu \mathrm{m}$. If the $h v^{2}$-concept is used here, one can have the same total $\mathrm{d} E$ while opening the exit slit to $200 \mu \mathrm{m}$ delivering $\delta E \simeq 480 \mathrm{meV}$. In simple terms, one can imagine that 20 horizontal stripes of the X-ray beam are laid vertically on the sample, with each stripe delivering a RIXS spectrum identical to the central one. Eventually, one sums up all of them into the total RIXS spectrum without loss of the total $\mathrm{d} E$. Thus the efficiency gain achieved by the imaging- $h v^{2}$ spectrometer by using the whole bandwidth of incident X-rays within the core-hole lifetime FWHM, even corrected for the additional mirrors in the optical scheme, is nearly a factor of 15 compared with the conventional RIXS instrumentation.

We note that in many cases such as orbital excitations the RIXS spectra are less sensitive to the $h v_{\text {in }}$-bandwidth. Furthermore, many experiments focus merely on energy and not on the magnitude or lineshape of the RIXS structures. In such cases the $h v^{2}$-concept allows utilization of incident photons through the whole energy extension of the multiplet and spin-orbit structure of the XAS spectrum, allowing much larger intensity gain compared with the core-lifetime window. This situation has a similarity to non-resonant X-ray fluorescence measurements, where one opens up the beamline exit slit to utilize incident photons in a large $h v_{\text {in }}$-window.

\section{Summary and outlook}

In summary, we have shown that the RIXS spectra as a function of energy loss are practically invariant to their integration over the $h v_{\text {in }}$-window equal to the core-hole lifetime. This fact permits the new generation of the RIXS instrumentation based on the $h v^{2}$-concept to utilize incident synchrotron radiation over the whole lifetime-wide bandwidth which is much larger than the required energy-loss resolution, thereby breaking the photon-count limit of the conventional RIXS instrumentation. At present the $h v^{2}$-concept is seen as the optimal way to keep RIXS going towards progressively refining the energy scale of charge-neutral excitations without any compromise on the precision and fullness of the spectroscopic information. A practical scheme of an imaging- $h v^{2}$ spectrometer tailored to modern diffraction-limited synchrotron sources will be published in our follow-up paper.

\section{Acknowledgements}

We thank Abhishek Nag for the support on the RIXS measurements, and Thorsten Schmitt for the fruitful discussions. We also acknowledge Diamond Light Source for providing science commissioning beam time at beamline I21.

\section{References}

Arpaia, R., Caprara, S., Fumagalli, R., De Vecchi, G., Peng, Y. Y., Andersson, E., Betto, D., De Luca, G. M., Brookes, N. B., Lombardi, F., Salluzzo, M., Braicovich, L., Di Castro, C., Grilli, M. \& Ghiringhelli, G. (2019). Science, 365, 906-910. 
Braicovich, L., van den Brink, J., Bisogni, V., Sala, M. M., Ament, L. J. P., Brookes, N. B., De Luca, G. M., Salluzzo, M., Schmitt, T., Strocov, V. N. \& Ghiringhelli, G. (2010). Phys. Rev. Lett. 104, 077002.

Chaix, L., Ghiringhelli, G., Peng, Y. Y., Hashimoto, M., Moritz, B., Kummer, K., Brookes, N. B., He, Y., Chen, S., Ishida, S., Yoshida, Y., Eisaki, H., Salluzzo, M., Braicovich, L., Shen, Z., Devereaux, T. P. \& Lee, W. (2017). Nat. Phys. 13, 952-956.

Chuang, Y., Anderson, C., Benk, M., Goldberg, K., Voronov, D., Warwick, T., Yashchuk, V. \& Padmore, H. A. (2016). AIP Conf. Proc. 1741, 050011.

Ghiringhelli, G., Le Tacon, M., Minola, M., Blanco-Canosa, S., Mazzoli, C., Brookes, N. B., De Luca, G. M., Frano, A., Hawthorn, D. G., He, F., Loew, T., Moretti Sala, M., Peets, D. C., Salluzzo, M., Schierle, E., Sutarto, R., Sawatzky, G. A., Weschke, E., Keimer, B. \& Braicovich, L. (2012). Science, 337, 821-825.

Ghiringhelli, G., Piazzalunga, A., Dallera, C., Trezzi, G., Braicovich, L., Schmitt, T., Strocov, V. N., Betemps, R., Patthey, L., Wang, X. \& Grioni, M. (2006). Rev. Sci. Instrum. 77, 113108.

Hepting, M., Chaix, L., Huang, E. W., Fumagalli, R., Peng, Y. Y., Moritz, B., Kummer, K., Brookes, N. B., Lee, W. C., Hashimoto, M., Sarkar, T., He, J. F., Rotundu, C. R., Lee, Y. S., Greene, R. L., Braicovich, L., Ghiringhelli, G., Shen, Z. X., Devereaux, T. P. \& Lee, W. S. (2018). Nature, 563, 374-378.
Krause, M. O. \& Oliver, J. H. (1979). J. Phys. Chem. Ref. Data, 8, 329338.

Lai, C. H., Fung, H. S., Wu, W. B., Huang, H. Y., Fu, H. W., Lin, S. W., Huang, S. W., Chiu, C. C., Wang, D. J., Huang, L. J., Tseng, T. C., Chung, S. C., Chen, C. T. \& Huang, D. J. (2014). J. Synchrotron Rad. 21, 325-332.

Le Tacon, M., Ghiringhelli, G., Chaloupka, J., Sala, M. M., Hinkov, V., Haverkort, M. W., Minola, M., Bakr, M., Zhou, K. J., BlancoCanosa, S., Monney, C., Song, Y. T., Sun, G. L., Lin, C. T., De Luca, G. M., Salluzzo, M., Khaliullin, G., Schmitt, T., Braicovich, L. \& Keimer, B. (2011). Nat. Phys. 7, 725-730.

Sarte, P. M., Songvilay, M., Pachoud, E., Ewings, R. A., Frost, C. D., Prabhakaran, D., Hong, K. H., Browne, A. J., Yamani, Z., Attfield, J. P., Rodriguez, E. E., Wilson, S. D. \& Stock, C. (2019). Phys. Rev. $B, \mathbf{1 0 0}, 075143$.

Schlappa, J., Wohlfeld, K., Zhou, K. J., Mourigal, M., Haverkort, M. W., Strocov, V. N., Hozoi, L., Monney, C., Nishimoto, S., Singh, S., Revcolevschi, A., Caux, J., Patthey, L., Rønnow, H. M., van den Brink, J. \& Schmitt, T. (2012). Nature, 485, 82-85.

Strocov, V. N. (2010). J. Synchrotron Rad. 17, 103-106.

Strocov, V. N., Schmitt, T., Flechsig, U., Patthey, L. \& Chiuzbăian, G. S. (2011). J. Synchrotron Rad. 18, 134-142.

Warwick, T., Chuang, Y.-D., Voronov, D. L. \& Padmore, H. A. (2014). J. Synchrotron Rad. 21, 736-743. 